\section{Transient retinal artery occlusion during phacoemulsification cataract surgery}

IH Yusuf, THM Fung, M Wasik and CK Patel

\begin{abstract}
Purpose Transient retinal artery occlusion (TRAO) is a potentially underdiagnosed cause of immediate 'pad off' visual loss following phacoemulsification cataract surgery under sub-Tenon's anaesthesia. Methods We describe a series of three patients presenting with enigmatic 'pad off' visual loss following phacoemulsification surgery, each diagnosed with TRAO. We describe the variable clinical presentation, illustrate the value of optical coherence tomography (OCT) imaging in establishing the diagnosis, and present the final visual outcomes.

Results Clinical findings alone may be

\section{Introduction}

Retinal artery occlusion is a rare, yet potentially devastating complication of phacoemulsification cataract surgery. ${ }^{1}$ We describe three patients presenting with acute post-operative visual loss following microincision phacoemulsification cataract surgery under sub-Tenon's anaesthesia ( $3 \mathrm{ml}$ of $2 \%$ lignocaine and 500 units of hyaluronate without adrenaline), each diagnosed with transient retinal artery occlusion (TRAO). All procedures were uncomplicated with a surgical duration of $<20$ min with standard infusion pressures. We describe the variable nature of clinical presentation and imaging studies in each case.
\end{abstract} subtle and inadequate in localising the pathology in patients with TRAO. Crosscomparison of superior and inferior macula OCT profiles in branch-pattern arterial occlusion-and between healthy and affected eyes in central-pattern arteriolar occlusion-is critical in clinching the diagnosis. The typical evolution of OCT appearance is acute-phase inner retinal thickening/oedema and hyperreflectivity followed by progressive, late-phase inner retinal atrophy. Visual acuity may recover but central scotomas, and defects in colour perception may persist.

Conclusion The diagnosis of TRAO is challenging; delayed presentation may resolve fundal and retinal angiographic abnormalities. OCT may be the only imaging modality that can provide objective evidence of TRAO. Meticulous comparison/ segmentation of OCT images is therefore mandatory in patients presenting with acute post-operative visual loss to exclude TRAO. Eye (2014) 28, 1375-1379; doi:10.1038/eye.2014.190; published online 8 August 2014
Case series

Case 1

A 74-year-old male presented on post-operative day 1 with visual acuity of counting fingers, improving to $6 / 18$ with a pinhole in the left eye. Fundal appearance was symmetrical and unremarkable, demonstrating mild age-related maculopathy with fine retinal epithelial pigmentary changes (Figures $1 \mathrm{a}$ and $\mathrm{b}$ ). Ten days later, vision improved to 6/18 (6/9 with a pinhole). There was no relative afferent pupillary defect. Paired OCT images 16 days post-operatively revealed subtle loss of laminar definition OS, noted only in retrospect, but retinal thickening was not present (Figures 1a and b). Fundus fluorescein (FFA) and indocyanine green angiography did not reveal any evidence of vascular occlusion. Visual field analysis demonstrated a perifoveal scotoma (Figure 1c). Improvement of visual acuity with pinhole suggested refractive error may be contributory: IOL exchange to an aspheric IOL
The Oxford Eye Hospital, West Wing, John Radcliffe Hospital, Oxford, UK

Correspondence: CK Patel, The Oxford Eye Hospital, West Wing, John Radcliffe Hospital, Headley Way, Headington, Oxford OX3 9DU, UK

Tel: +44 (0)1865 234736; Fax: +44 (0)1865 234736 .

E-mail: ckpatel@

btinternet.com

Received: 25 February 2014 Accepted in revised form: 3 July 2014

Published online:

8 August 2014 
a

b

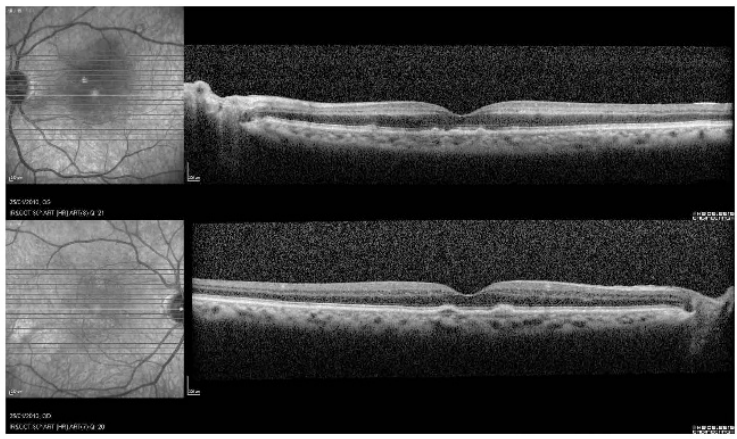

c

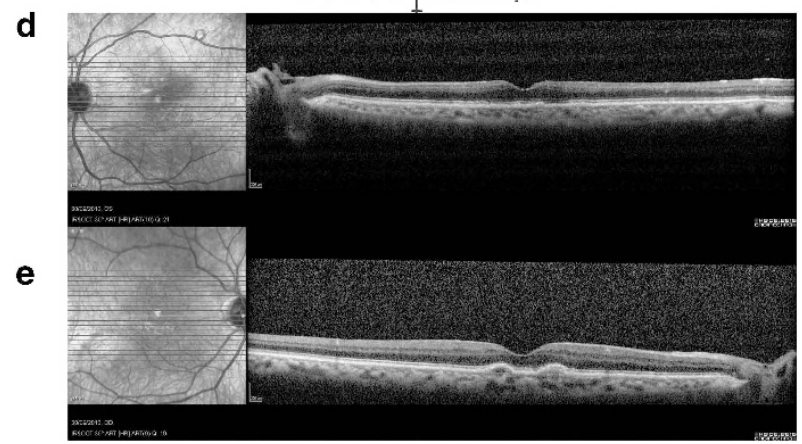

Figure 1 ( $a$ and $b$ ) Paired OCT images demonstrating subtle loss of laminar differentiation of the left inner retina when compared with the right. Three-dimensional (3D) volumetric segmentation analysis revealed symmetrical inner retinal volumes of $3.98 \mathrm{~mm}^{3}$ OS and $4.11 \mathrm{~mm}^{3}$ OD (retinal thickness measured from internal limiting membrane to the inner nuclear layer across a standard 20。 macula field using Heidelberg Eye Explorer). There is no cystoid macular oedema but retinal pigment epithelium folds are seen bilaterally. (c) 10-2 automated visual field analysis revealed left central scotoma. (d and e) OCT images one year later demonstrate diffuse left inner retinal thinning when compared with the right eye, and with the same eye at presentation (3D volumetric segmentation analysis: $3.13 \mathrm{~mm}^{3}$ OS compared with $4.05 \mathrm{~mm}^{3} \mathrm{OD}$ ) with further loss of laminar differentiation.

lens was undertaken and photorefractive keratectomy thereafter to eliminate remaining cylindrical error. However, visual acuity remained at $6 / 18$ OS (6/9 with a pinhole). Farnsworth-Munsell 100 hue revealed poor colour discrimination OS. Nine months later, left inner retinal atrophy was evident confirming the diagnosis of
TRAO (Figures 1d and e). A carotid ultrasonography and cardiac investigations did not reveal any abnormalities.

\section{Case 2}

A 46-year-old male presented on post-operative day 1 with a right superior scotoma and visual acuity of $6 / 9$ OD. There was no cardiovascular history of note at presentation. Dilated fundus examination revealed inferior macular opacification but no embolus (Figure 2a). FFA revealed normal retinal arterial and retinal capillary perfusion (Figure $2 b$ ). Visual field assessment revealed a dense superior scotoma, extending inferotemporally (Figure 2c). OCT demonstrated asymmetric right inferior inner retinal thickening, not present superiorly (Figures $2 \mathrm{~d}$ and e). One month later, visual acuity was $6 / 5$ OD with persistent visual field defect, although retinal appearance had normalised. Echocardiography revealed a patent foramen ovale, which was closed on cardiac catheterisation. Two years later, vision remained at $6 / 5$, although OCT revealed right inferior inner retinal atrophy (Figure 2f).

\section{Case 3}

A 52-year-old male presented on post-operative day 4 with a left-sided central, circular scotoma noted in the immediate post-operative period. He had known atrial fibrillation for which he took sotalol and warfarin daily (international normalised ratio was 2.7 at the time of surgery). Fundal examination revealed a cherry-red spot at the fovea. FFA demonstrated perifoveal hypoperfusion, although no discrete arterial occlusion was seen (Figure 3a). Seven sessions of hyperbaric oxygen therapy were undertaken in the first postoperative month, starting on post-operative day 7. OCT revealed diffuse inner retinal thickening (Figure $3 b$ ), followed by inner retinal atrophy 1 year later (Figure 3c). Vision improved over a 2-year period to $6 / 6$ with reduced red saturations.

\section{Discussion}

TRAO is a potentially underdiagnosed cause of acute post-operative visual loss in patients undergoing nontopical phacoemulsification cataract surgery under local anaesthesia (sub-Tenon's, peribulbar, and retrobulbar). ${ }^{1}$ The incidence of TRAO has not been evaluated in prospective longitudinal case finding studies, although it can be considered as a rare event. ${ }^{1}$ We present the largest case series reported of TRAO following phacoemulsification under sub-Tenon's anaesthesia.

TRAO is likely to represent a clinical continuum: clinical features such as reduced acuity, visual field loss, 


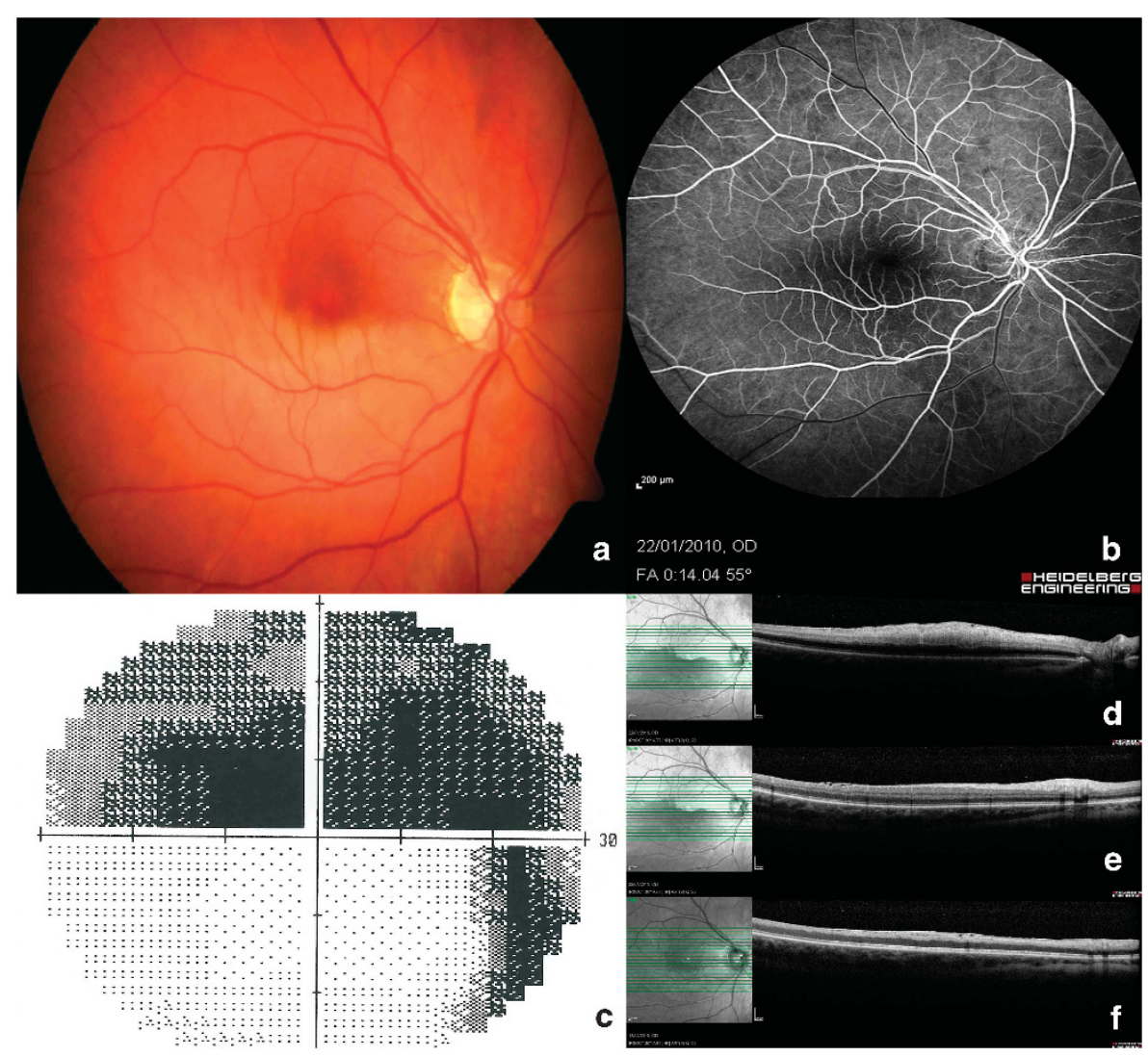

Figure 2 (a) Right digital fundus photograph demonstrates inferior retinal whitening/opacification suggestive of oedema although no embolus is seen. (b) Mid-venous fluorescein fundus angiogram image demonstrates normal retinal arterial tree and absence of vascular occlusion. (c) Right 30-2 automated visual field analysis reveals dense corresponding superior visual field loss extending inferotemporally. (d) Inferior macular OCT demonstrates gross, diffuse inner retinal thickening with loss of laminar differentiation (3D volumetric segmentation analysis: inner retinal volume of inferior macula hemifield $=5.22 \mathrm{~mm}^{3}$ ). (e) Superior macula OCT profile demonstrates normal laminar retinal differentiation (3D volumetric segmentation analysis: inner retinal volume of superior macula hemifield $=3.63 \mathrm{~mm}^{3}$ ). (f) One year post-operatively, inner retinal atrophy is demonstrated (3D volumetric segmentation analysis: inner retinal thickness of inferior macula hemifield $=2.59 \mathrm{~mm}^{3}$ ).

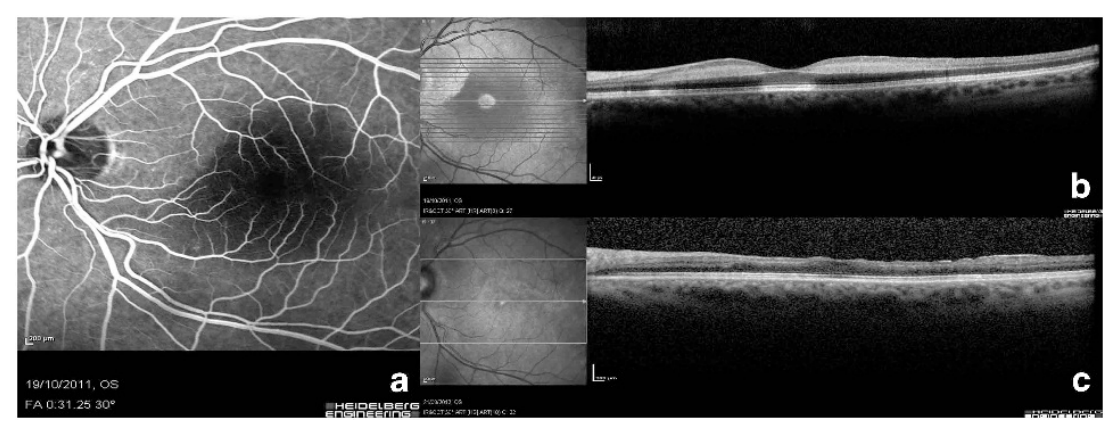

Figure 3 (a) Mid-venous FFA revealing diffuse macular hypoperfusion demonstrated by perifoveal macular hypofluorescence. There is no leakage suggestive of sub-clinical cystoid macular oedema. (b) Acute inner retinal thickening at presentation (3D volumetric segmentation analysis: inner retinal volume $=5.16 \mathrm{~mm}^{3}$ ). (c) One year post-operatively, gross inner retinal atrophy is demonstrated $(3 \mathrm{D}$ volumetric segmentation analysis: inner retinal volume $3.02 \mathrm{~mm}^{3}$ ).

and colour deficits may variably be present, ${ }^{2,3}$ influenced by the anatomical location, extent and duration of vascular occlusion, or spasm. ${ }^{4,5}$ A duration of anoxia of
$>4 \mathrm{~h}$ exceeds the retina's maximal survival time, ${ }^{6}$ although neuronal cell death may occur within $97 \mathrm{~min}^{7}$ TRAO of very short duration may not produce visual 
symptoms, ${ }^{1}$ and further investigation will not be sought. An intermediate duration of occlusion will result in symptoms ${ }^{1,3,8}$ despite arteriolar reperfusion proven on FFA; these patients are labelled as having suffered TRAO. ${ }^{1}$

TRAO may be easily overlooked in the post-operative period. Patients who experience mild early symptoms may not consider them significant, or another clinical finding may be offered by the ophthalmologist (refractive error, post-operative inflammation, and so on) to explain them. Patients with persistent symptoms (reduced visual acuity, colour perception, or field loss) may undergo OCT specifically to exclude foveal cystic macular oedema or FFA to exclude arterial occlusion-neither of which will correctly identify TRAO. Visual acuity improving with a pinhole correction may be present in patients with TRAO, perhaps secondary to a diffractive phenomenon consequent to inner retinal oedema. This may erroneously direct diagnostic thinking towards a refractive aetiology of visual loss and delay the diagnosis of TRAO (case 1).

OCT imaging may provide the only objective evidence of TRAO, particularly in patients not presenting in the immediate post-operative period. The acute phase OCT imaging may reveal increased thickness and hyperreflectivity of the inner retina secondary to oedema from the ischaemic event. ${ }^{9-11}$ This may persist for up to 1 month and previously demonstrated maximal on day $3 .{ }^{12}$ The inner plexiform layer demonstrates the greatest net change in thickness. ${ }^{10}$ OCT evidence of inner retinal atrophy occurs 60-90 days after retinal artery occlusion. ${ }^{9-12}$ OCT examination after several weeks may not reveal retinal thickening owing to the resolution of oedema, before the onset of retinal atrophy (case 1).

OCT findings are subtle and meticulous crosscomparison must be undertaken to diagnose TRAO; there are no easily identifiable cystic changes. 'Branchpattern' TRAO may reveal asymmetry of inner retinal appearance between the superior and inferior macula (case 2). 'Central-pattern' TRAO may reveal inner retinal asymmetry between right and left eyes (cases 1 and 3). Segmentation volumetric analysis of OCT images may add objective measures of inner retinal thickness to document acute-phase oedema and late-phase atrophy, supporting a diagnosis of TRAO. ${ }^{10}$ Identification of TRAO is difficult: case 1 was diagnosed retrospectively on re-examination of OCT images following our experience with cases 2 and 3 .

The mechanism of TRAO following phacoemulsification cataract surgery in this series remains unclear. The small volumes of sub-Tenon's lignocaine without adrenaline used suggest that raised orbital and intraocular pressure exceeding mean arterial pressure, or pharmacological vasoconstriction unlikely. No other systemic or ocular parameter was identified as contributory, although a prospective case-finding study is required to identify risk factors. We therefore suggest that TRAO may be an idiosyncratic occurrence.

Sub-Tenon's anaesthesia is considered safe, but it is possible that even small volumes may contribute to the risk of TRAO; use of topical and intracameral anaesthetic may conceivably reduce the risk of TRAO. Augmentation with subconjunctival lignocaine adjacent to corneal wounds may reduce perception of surgical stimuli for less co-operative patients.

Post-operative TRAO is a difficult diagnosis. Ophthalmologists must be aware of TRAO as a cause of 'pad off' visual loss following phacoemulsification surgery and meticulously cross-compare inner retinal appearance on OCT imaging between healthy and affected eyes.

\section{Summary}

What was known before

- Transient retinal artery occlusion is a potentially underdiagnosed cause of acute visual loss following phacoemulsification cataract surgery under sub-Tenon's anaesthesia. Fundus fluorescein angiography may not demonstrate abnormalities due to reperfusion of the transiently occluded retinal vessel.

What this study adds

- Optical coherence tomography (OCT) may demonstrate the only objective signs of transient retinal artery occlusion. Acute presentation may demonstrate OCT evidence of inner retinal thickening/hyperreflectivity. Late presentation may reveal generalised inner retinal atrophy in the distribution of the occluded artery.

\section{Conflict of interest}

The authors declare no conflict of interest.

\section{References}

1 Feibel RM, Guyton DL. Transient central retinal artery occlusion after posterior sub-Tenon's anesthesia. J Cataract Refract Surg 2003; 29(9): 1821-1824.

2 Ascaso FJ. Transient central retinal artery occlusion following peribulbar anesthesia for pars plana vitrectomy. J Clin Anesth 2010; 22(7): 577-578.

3 Chalam KV, Agarwal S, Gupta SK, Shah GY. Recovery of retinal sensitivity after transient branch retinal artery occlusion. Ophthalmic Surg Lasers Imaging 2007; 38(4): 328-329.

4 Hayreh SS. Vasospasm and transient monocular blindness. N Engl J Med 1992; 326(12): 837-838.

5 Burger SK, Saul RF, Selhorst JB, Thurston SE. Transient monocular blindness caused by vasospasm. $N$ Engl J Med 1991; 325(12): 870-873.

6 Hayreh SS, Zimmerman MB, Kimura A, Sanon A. Central retinal artery occlusion. Retinal survival time. Exp Eye Res 2004; 78(3): 723-736. 
7 Hayreh SS. Acute retinal arterial occlusive disorders. Prog Retin Eye Res 2011; 30(5): 359-394.

8 Hayreh SS. Transient central retinal artery occlusion following viperine snake bite. Arch Ophthalmol 2008; 126(6): 870-871.

9 Chu YK, Hong YT, Byeon SH, Kwon OW. In vivo detection of acute ischemic damages in retinal arterial occlusion with optical coherence tomography: a 'prominent middle limiting membrane sign'. Retina 2013; 33(10): 2110-2117.
10 Ritter M, Sacu S, Deak GG, Kircher K, Sayegh RG, Pruente C et al. In vivo identification of alteration of inner neurosensory layers in branch retinal artery occlusion. Br J Ophthalmol 2012; 96(2): 201-207.

11 Ikeda F, Kishi S. Inner neural retina loss in central retinal artery occlusion. Jpn J Ophthalmol 2010; 54(5): 423-429.

12 Saxena S, Mishra N, Meyer CH, Akduman L. Ischaemiareperfusion injury in central retinal artery occlusion. BMJ Case Rep 2013; e-pub ahead of print 21 October 2013; doi:10.1136/bcr-2013-201415. 